\title{
Oxidative stress induced by portal vein embolization in fatty liver: Experimental study of a nonalcoholic steatohepatitis model
}

\author{
YASUTAKA BABA $^{1,2}$, SADAO HAYASHI $^{1}$, KOHEI NAGASATO $^{1}$, MICHIYO HIGASHI $^{3}$, \\ NANAKO TOSUJI ${ }^{4}$, SHUNRO SONODA ${ }^{4}$ and TAKASHI YOSHIURA ${ }^{1}$ \\ ${ }^{1}$ Department of Radiology, Graduate School of Medical and Dental Sciences, Kagoshima University, Kagoshima 890-8544; \\ ${ }^{2}$ Department of Diagnostic Radiology, Hiroshima University, Minami-ku, Hiroshima 734-8551; \\ Departments of ${ }^{3}$ Human Pathology, and ${ }^{4}$ International Island and Community Medicine, \\ Graduate School of Medical and Dental Sciences, Kagoshima University, Kagoshima 890-8544, Japan
}

Received January 8, 2018; Accepted August 7, 2018

DOI: $10.3892 /$ br.2018.1141

\begin{abstract}
The present study aimed to investigate whether excessive oxidative stress production or reduction of antioxidative stress potential may occur following portal vein embolization (PVE) in an experimental animal nonalcoholic steatohepatitis (NASH) model. A NASH rabbit model $(n=11)$ was established by feeding of a fat diet for 4 weeks, and a normal diet rabbit model $(n=11)$ was prepared as a control. The oxidative status of NASH was examined by measuring derivatives of reactive oxygen metabolites (d-ROM) for oxidative stress and biological antioxidative potential (BAP) for antioxidative potential in the NASH model and normal group. Additionally, oxidative status of PVE after 2 weeks was assessed by measuring d-ROM and BAP in the NASH and normal liver models. Oxidative status in a PVE+NASH model was also detected. In the process of NASH creation (fat diet for 4 weeks), total cholesterol was increased in the NASH group $(\mathrm{P}<0.0001)$. In the NASH group, $\mathrm{PVE}$ induced an increase in serum aspartate transaminase $(\mathrm{P}=0.0318)$. At 4 weeks after initiation of the fat diet, a decrease in BAP was determined as statistically significant $(\mathrm{P}<0.0001)$. In normal liver, d-ROM production was stimulated in the Sham group after 2 weeks $(\mathrm{P}=0.0152)$, but BAP was not altered $(\mathrm{P}=0.6119)$. In NASH liver, d-ROM production was stimulated in PVE and Sham groups $(\mathrm{P}<0.0001$ and $\mathrm{P}=0.0189$, respectively), but $\mathrm{BAP} \operatorname{did}$
\end{abstract}

Correspondence to: Dr Yasutaka Baba, Department of Diagnostic Radiology, Hiroshima University, 1-2-3 Kasumi, Minami-ku, Hiroshima 34-8551, Japan

E-mail: ybaba@hiroshima-u.ac.jp

Abbreviations: NASH, nonalcoholic steatohepatitis; SOS, sinusoid obstruction syndrome; d-ROM, derivatives of reactive oxygen metabolites; BAP, biological antioxidative potential; PVE, portal vein embolization; AST aspartate transaminase; ALT, alanine aminotransferase; TG, triglyceride; T-Chol, total cholesterol; NAS, nonalcoholic fatty liver disease activity score

Key words: oxidative stress, antioxidant potential, nonalcoholic steatohepatitis, portal vein embolization not change $(\mathrm{P}>0.05)$. In conclusion, decrease of antioxidant potential may promote NASH progression. Additionally, PVE appeared to cause a surge in oxidative stress in NASH liver.

\section{Introduction}

Chronic inflammation is causatively associated with liver cancer as previously documented by our group (1). Our group also reported a possibility of increased oxidative stress induced by use of anticancer drugs in patients with hepatocellular carcinoma (1). Therefore, in the clinic there should be consideration of the potential for excessive oxidative stress during anticancer treatment in cancer patients.

In order to prevent liver failure following liver surgery in patients with primary and secondary liver malignancy, portal vein embolization (PVE) is the widely accepted strategy for increasing the volume of the liver segments that will remain following liver lobe resection (2). Although portal vein embolization is considered a safe technique, there is a potential risk of liver failure associated with nonalcoholic steatohepatitis (NASH) (3) and sinusoid obstruction syndrome (SOS) following systemic chemotherapy (4).

Narita et al (4) reported that SOS inhibited future remnant liver hypertrophy following PVE and induced postoperative liver failure. As the indication of extended hepatectomy is expanded in patients with chronic liver damage (NASH) or metastatic liver cancer following repeated systemic chemotherapy, liver damage should be considered when PVE is intended (4).

To evaluate a pathological role of oxidative stress associated with inflammatory fatty liver, the current study investigated biological anti-oxidative potential (BAP) and derivatives of reactive oxygen metabolites (d-ROM) using plasma samples from rabbits raised on fatty diets. Via PVE pretreatment, reproducible measurements of BAP and d-ROM were enabled in the white-colored plasma, and endogenous oxidative stress in the fatty diet animal model was confirmed.

The current study presents a novel approach to gaining insight into lipid metabolism and associated liver cancer by measuring BAP and d-ROM as biomarkers of oxidative stress in fatty liver and of the malignant process in liver cancers. The 
purpose of this study was to investigate whether PVE has an influence on liver damage in an NASH model.

\section{Materials and methods}

Experimental schedule. The schedule of experimental study is depicted in Figs. 1 and 2. A total of 22 male New Zealand rabbits [KBT Oriental Co., Ltd., Saga, Japan; mean weight $2.5 \mathrm{~kg}$ (range 1.9-2.7 kg)] were used. The rabbits were housed at $23+/-1^{\circ} \mathrm{C}$ under a $12-\mathrm{h}$ light/dark cycle (lights on at 7 a.m.) and $40-70 \%$ atmosphere, and were fed $100 \mathrm{~g}$ standard diet (LRC4: Oriental Yeast Co., Ltd., Kyoto, Japan) once per day and provided water ad libitum. The rabbits were divided into two groups ( $n=11 /$ group): Normal diet and fat diet (NASH group). In the normal diet group, two subgroups were established as PVE $(n=6)$ and Sham $(n=5)$ (Fig. 1). In the fat diet group, PVE $(n=5)$ and Sham $(n=6)$ subgroups were established at 4 weeks after feeding a fat diet (Fig. 2).

All procedures were performed under general anesthesia. Animals were placed supine, and then general anesthesia was induced with intramuscular injection of a mixture of ketamine hydrochloride $(25 \mathrm{mg} / \mathrm{kg}$ Ketalar Intramuscular $500 \mathrm{mg}$; Daiichi Sankyo Co., Ltd., Tokyo, Japan) and medetomidine chloride (0.5 mg/kg Domitor; Zenoaq, Fukushima, Japan), then maintained with administration of isoflurane (3\% Isoflu; DS Pharma Animal Health Co., Ltd., Osaka, Japan) through a mask. Oxygen saturation was monitored using a pulse oximeter (BSM-5105; Nihon Koden, Tokyo, Japan). The study protocol was approved by the Institutional Animal Care and Use Committee of Kagoshima University (Kagoshima, Japan; approval no. MD11117).

$P V E$. Under deep anesthesia and using a fluoroscopic unit (TU-50; Hitachi Ltd., Tokyo, Japan), PVE was performed in the left lobe of each PVE group using $1 \mathrm{ml}$ absolute ethanol (5). Following open laparotomy, the mesenteric vein was harvested and a microcatheter (Snyper 2; Terumo Corporation, Tokyo, Japan) was inserted and advanced to the left lateral portal vein. After verifying placement of the microcatheter in the lateral left lobe using a small amount $(1 \mathrm{ml})$ of contrast medium (Omnipaque 300; Bayer Holding Ltd., Tokyo, Japan), $1 \mathrm{ml}$ absolute ethanol was injected and a 3- or 4-mm-diameter Vortex microcoil was positioned in the lateral left portal vein (Boston Scientific Japan K.K., Tokyo, Japan). Following PVE, it was confirmed that the left portal vein branch had disappeared and the portal vein main trunk was dilated. Rabbits were sacrificed 2 weeks after PVE. For the Sham group, open laparotomy was performed and the suture was closed without any other interventions.

NASH model preparation. Rabbits were fed a high-fat diet (standard diet $+10 \%$ lard $+2 \%$ cholesterol) for 4 weeks (6). After feeding the fat diet for 4 weeks, a normal diet was resumed to avoid unintentional liver damage.

Measurement of oxidative stress (d-ROM and BAP). Measurements of d-ROM and BAP were performed as previously reported (1). Freshly drawn and heparinized peripheral blood $(3 \mathrm{ml})$ was spun in a low-speed centrifuge to separate the plasma. Measurements of d-ROM and BAP in plasma

$$
\text { Normal liver + PVE }(n=6)
$$
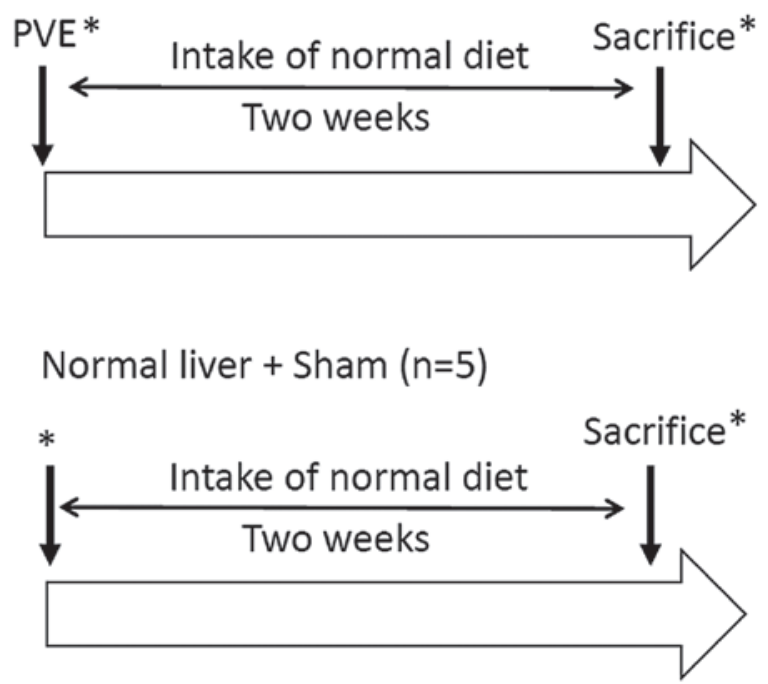

Figure 1. Flowchart of study protocol for the normal liver group. *Venous blood sampling. PVE, portal vein embolization.

were performed within 2 h using a Free Radical Analytical System 4 (FRAS4) designed by SEAC s.r.l. (Florence, Italy). For the measurement of d-ROM, $10 \mu \mathrm{l}$ fresh plasma was mixed with $1 \mathrm{ml}$ of a reagent containing $\mathrm{FeCl}_{2}\left(\mathrm{Fe}^{2+}\right)$ and diethyl p-phenylene diamine chromogen, then incubated for $5 \mathrm{~min}$ at $37^{\circ} \mathrm{C}$ to develop the oxidized color. The change in optical density at $505 \mathrm{~nm}$ was measured over the 5-min reaction time. For the measurement of BAP, $1 \mathrm{ml}$ of an oxyradical colorant comprising a thiocyanate compound and $\mathrm{FeCl}_{3}\left(\mathrm{Fe}^{3+}\right)$ was reduced by the addition of $10 \mu \mathrm{l}$ of the rabbit plasma, and the decrease in optical density at $505 \mathrm{~nm}$ over the 5-min reaction time at $37^{\circ} \mathrm{C}$ was measured in the FRAS4 according to the manufacturer's instructions. d-ROM and BAP readings were taken for each sample and used to assess the level of biological oxidative stress.

As rabbits produced a chylous plasma, for which the milky turbidity interfered with the optical densitometry in measurement of BAP and d-ROM, all milky plasma had to be clarified by skimming fatty components with petroleum ether (PE) prior to ordinary assay of BAP and d-ROM in the FRAS4. Prior to pathological assessment of NASH, it was clarified whether oxidative stress markers differed between PVE and Sham groups in normal and NASH rabbit models.

Laboratory assessment. Laboratory data including serum aspartate transaminase (AST), alanine aminotransferase (ALT), triglyceride (TG) and total cholesterol (T-Chol) in the normal and NASH groups were also checked. After the experimental procedure, all rabbits were sacrificed and pathological specimens of the non-embolized liver lobe were investigated for the presence, grade and score of NASH.

NASH diagnosis. Pathological diagnosis was assessed according to the classification of NASH reported by Brunt et al (7), with grade evaluated by dividing lesions into active and potentially reversible injury according to Nonalcoholic fatty liver disease Activity Score (NAS). The 
Table I. Laboratory data in the normal liver group.

\begin{tabular}{|c|c|c|c|c|c|c|c|c|c|c|}
\hline & \multicolumn{2}{|c|}{ AST } & \multicolumn{2}{|c|}{ ALT } & \multicolumn{2}{|c|}{ TG } & \multicolumn{2}{|c|}{ T-Chol } & \multicolumn{2}{|c|}{ ALT/AST } \\
\hline & $0 \mathrm{~W}$ & $2 \mathrm{~W}$ & $0 \mathrm{~W}$ & $2 \mathrm{~W}$ & $0 \mathrm{~W}$ & $2 \mathrm{~W}$ & $0 \mathrm{~W}$ & $2 \mathrm{~W}$ & $0 \mathrm{~W}$ & $2 \mathrm{~W}$ \\
\hline \multicolumn{11}{|c|}{ Normal + PVE $(n=6)$} \\
\hline Mean & 31.8 & 36.8 & 45.0 & 45.8 & 41.3 & 58.1 & 43.6 & 121.6 & 1.63 & 1.64 \\
\hline $\mathrm{SD}$ & 12.0 & 26.5 & 26.0 & 26.4 & 18.0 & 36.4 & 25.0 & 64.9 & 1.05 & 0.79 \\
\hline $\mathrm{n}$ & 6 & 6 & 6 & 6 & 6 & 6 & 6 & 6 & 6 & 6 \\
\hline P-value & \multicolumn{2}{|c|}{0.6827} & \multicolumn{2}{|c|}{0.9589} & \multicolumn{2}{|c|}{0.3348} & \multicolumn{2}{|c|}{0.0206} & \multicolumn{2}{|c|}{0.9864} \\
\hline \multicolumn{11}{|c|}{ Normal + Sham $(n=5)$} \\
\hline Mean & 28.6 & 14.8 & 66.2 & 32.4 & 34.6 & 44.0 & 36.4 & 35.0 & 2.40 & 2.40 \\
\hline SD & 9.30 & 7.70 & 20.5 & 10.8 & 18.5 & 12.8 & 13.0 & 9.60 & 0.70 & 0.80 \\
\hline $\mathrm{n}$ & 5 & 5 & 5 & 5 & 5 & 5 & 5 & 5 & 5 & 5 \\
\hline P-value & \multicolumn{2}{|c|}{0.0339} & \multicolumn{2}{|c|}{0.0115} & \multicolumn{2}{|c|}{0.3775} & \multicolumn{2}{|c|}{0.8512} & \multicolumn{2}{|c|}{1} \\
\hline
\end{tabular}

PVE, portal vein embolization; AST aspartate transaminase; ALT, alanine aminotransferase; TG, triglyceride; T-Chol, total cholesterol; W, weeks; SD, standard deviation.
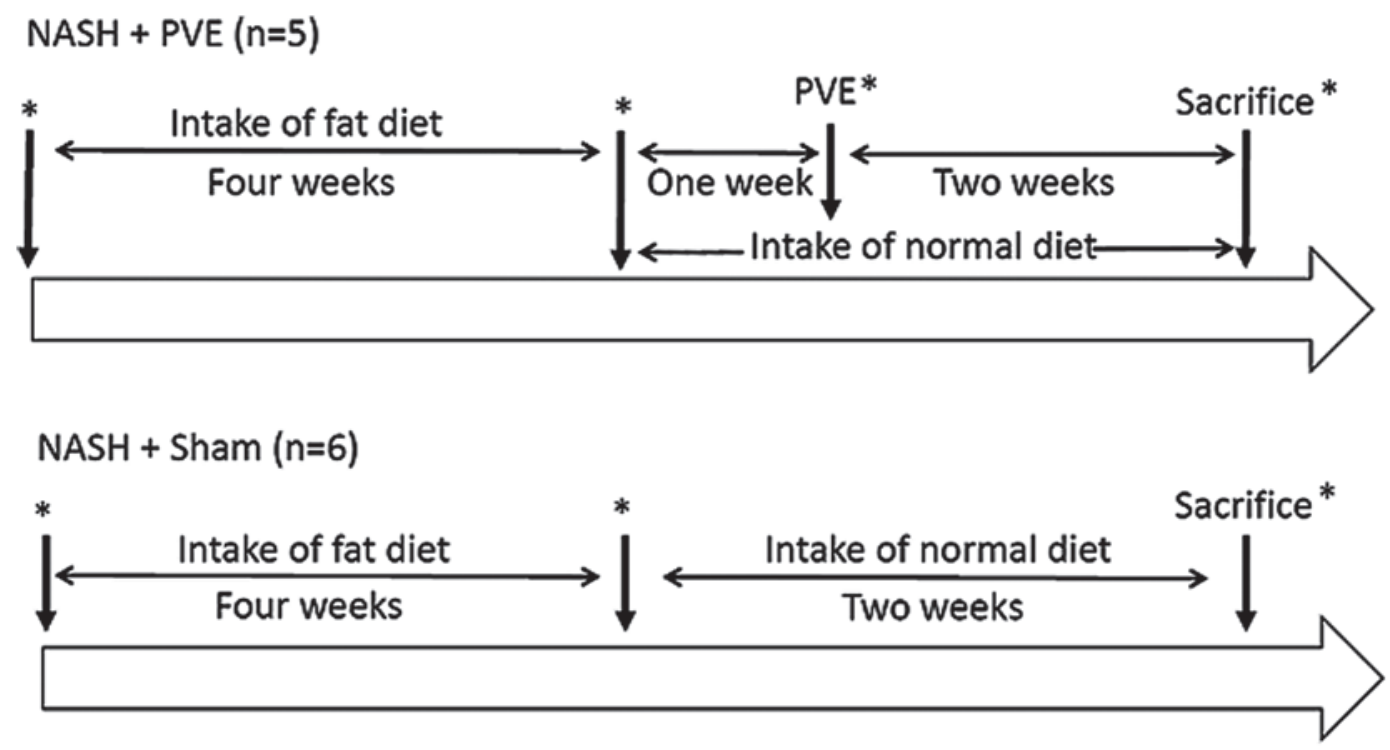

Figure 2. Study flowchart for the NASH liver group. "Venous blood sampling. NASH, nonalcoholic steatohepatitis.

proposed NAS also separates three grades: Steatosis, lobular inflammation, and ballooning. Individual features were graded semiquantitatively according to the scale: 0 , absent; 1 , mild; 2, moderate; and 3, severe. Severity of steatosis was based on the proportion of hepatocytes involved: $1,<33 \% ; 2,33-66 \%$; $3,>66 \%$. Severity of lobular inflammation was based on the number of inflammatory foci per $\times 20$ field: Score 1, 1-2 foci; score 2, 2-4 foci; score 3,>4 foci. Stage was evaluated as potentially less reversible and characterized by collagen deposition and architectural alterations that may evolve toward more permanent parenchymal remodeling.

Statistical analysis. Statistical analysis was performed using Student's t-test for continuous variables of paired data. The Mann-Whitney $U$ test was used for continuous variables of unpaired data and the $\chi^{2}$ test for categorical variables of unpaired data. MedCalc version 9.5.1.0 software (MedCalc Software bvba, Ostend, Belgium) was used for all analyses. A two-tailed value of $\mathrm{P}<0.05$ was considered to indicate a statistically significant result.

\section{Results}

Laboratory results of PVE and Sham treatments in the NASH and normal liver models. Laboratory data are summarized in Tables I and II. A variable effect was observed on laboratory parameters when comparing the PVE and Sham treatment groups [PVE group: AST was marginally increased, ALT was relatively unchanged, TG was increased and total cholesterol was markedly increased $(\mathrm{P}=0.0206)$; Table I $]$. In the process of NASH creation (fat diet for 4 weeks), T-Chol was markedly increased in the NASH group $(\mathrm{P}<0.0001)$. In the NASH group, PVE induced 
Table II. Laboratory data in the NASH liver group.

\begin{tabular}{|c|c|c|c|c|c|c|c|c|c|c|}
\hline & \multicolumn{2}{|c|}{ AST } & \multicolumn{2}{|c|}{ ALT } & \multicolumn{2}{|c|}{$\mathrm{TG}$} & \multicolumn{2}{|c|}{ T-Chol } & \multicolumn{2}{|c|}{ ALT/AST } \\
\hline & ow & $4 \mathrm{~W}$ & oW & $4 \mathrm{~W}$ & ow & $4 \mathrm{~W}$ & oW & $4 \mathrm{~W}$ & oW & $4 \mathrm{~W}$ \\
\hline \multicolumn{11}{|c|}{ Total NASH animals $(n=11)$} \\
\hline Mean & 23.9 & 32.6 & 51.4 & 49.2 & 50.3 & 146.6 & 48.6 & $3,431.9$ & 2.26 & 1.63 \\
\hline SD & 12.1 & 13.1 & 31.6 & 19.6 & 11.5 & 172.3 & 24.7 & $1,092.4$ & 1.07 & 0.60 \\
\hline $\mathrm{n}$ & 11 & 11 & 11 & 11 & 11 & 11 & 11 & 11 & 11 & 11 \\
\hline \multirow[t]{3}{*}{ P-value } & \multicolumn{2}{|c|}{0.1026} & \multicolumn{2}{|c|}{0.8395} & \multicolumn{2}{|c|}{0.0664} & \multicolumn{2}{|c|}{$<0.0001$} & \multicolumn{2}{|c|}{0.0885} \\
\hline & \multicolumn{2}{|c|}{ AST } & \multicolumn{2}{|c|}{ ALT } & \multicolumn{2}{|c|}{ TG } & \multicolumn{2}{|c|}{ T-Chol } & \multicolumn{2}{|c|}{ ALT/AST } \\
\hline & $5 \mathrm{~W}$ & $7 \mathrm{~W}$ & $5 \mathrm{~W}$ & $7 \mathrm{~W}$ & $5 \mathrm{~W}$ & $7 \mathrm{~W}$ & $5 \mathrm{~W}$ & $7 \mathrm{~W}$ & $5 \mathrm{~W}$ & $7 \mathrm{~W}$ \\
\hline \multicolumn{11}{|c|}{$\mathrm{NASH}+\mathrm{PVE}(\mathrm{n}=5)$} \\
\hline Mean & 48.2 & 78.6 & 36.6 & 55.6 & 146.8 & 136.8 & $3,603.4$ & 2169.8 & 0.80 & 0.70 \\
\hline SD & 8.10 & 24.9 & 8.20 & 16.7 & 45.2 & 59.3 & $1,327.4$ & 500.0 & 0.20 & 0.20 \\
\hline $\mathrm{n}$ & 5 & 5 & 5 & 5 & 5 & 5 & 5 & 5 & 5 & 5 \\
\hline \multirow[t]{3}{*}{ P-value } & \multicolumn{2}{|c|}{0.0318} & \multicolumn{2}{|c|}{0.0518} & \multicolumn{2}{|c|}{0.7719} & \multicolumn{2}{|c|}{0.0537} & \multicolumn{2}{|c|}{0.452} \\
\hline & \multicolumn{2}{|c|}{ AST } & \multicolumn{2}{|c|}{ ALT } & \multicolumn{2}{|c|}{ TG } & \multicolumn{2}{|c|}{ T-Chol } & \multicolumn{2}{|c|}{ ALT/AST } \\
\hline & $4 \mathrm{~W}$ & $6 \mathrm{~W}$ & $4 \mathrm{~W}$ & $6 \mathrm{~W}$ & $4 \mathrm{~W}$ & $6 \mathrm{~W}$ & $4 \mathrm{~W}$ & $6 \mathrm{~W}$ & $4 \mathrm{~W}$ & $6 \mathrm{~W}$ \\
\hline \multicolumn{11}{|c|}{ NASH+SHAM (n=6) } \\
\hline Mean & 33.0 & 33.7 & 58.3 & 41.9 & 56.7 & 63.9 & $3,032.4$ & 2163 & 1.84 & 1.35 \\
\hline $\mathrm{SD}$ & 12.1 & 7.40 & 19.4 & 10.5 & 54.4 & 41.5 & 1,097 & 622.3 & 0.37 & 0.54 \\
\hline $\mathrm{n}$ & 6 & 6 & 6 & 6 & 6 & 6 & 6 & 6 & 6 & 6 \\
\hline P-value & \multicolumn{2}{|c|}{0.8983} & \multicolumn{2}{|c|}{0.0717} & \multicolumn{2}{|c|}{0.7879} & & 932 & & 425 \\
\hline
\end{tabular}

NASH, nonalcoholic steatohepatitis; PVE, portal vein embolization; AST aspartate transaminase; ALT, alanine aminotransferase; TG, triglyceride; T-Chol, total cholesterol; W, weeks; SD, standard deviation.

Table III. Histological findings in NASH model and normal liver groups.

\begin{tabular}{|c|c|c|c|c|c|c|}
\hline Factor & Group & None & Mild & Moderate & Severe & P-value \\
\hline \multirow[t]{2}{*}{ Steatosis } & NASH model $(n=6)$ & 0 & 0 & 0 & 6 & \multirow[t]{2}{*}{0.0011} \\
\hline & Normal liver $(n=7)$ & 7 & 0 & 0 & 0 & \\
\hline \multirow[t]{2}{*}{ Ballooning } & NASH model $(n=6)$ & 0 & 0 & 0 & 6 & \multirow[t]{2}{*}{0.0019} \\
\hline & Normal liver $(\mathrm{n}=7)$ & 4 & 3 & 0 & 0 & \\
\hline \multirow[t]{2}{*}{ Inflammation } & NASH model $(n=6)$ & 0 & 1 & 5 & 0 & \multirow[t]{2}{*}{0.0032} \\
\hline & Normal liver $(\mathrm{n}=7)$ & 5 & 2 & 0 & 0 & \\
\hline \multirow[t]{2}{*}{ NASH stage } & NASH model $(n=6)$ & 0 & 6 & 0 & 0 & \multirow[t]{2}{*}{0.0011} \\
\hline & Normal liver $(n=7)$ & 7 & 0 & 0 & 0 & \\
\hline \multirow[t]{2}{*}{ NASH grade } & NASH model $(n=6)$ & 0 & 0 & 0 & 6 & \multirow[t]{2}{*}{0.0005} \\
\hline & Normal liver $(n=7)$ & 0 & 7 & 0 & 0 & \\
\hline
\end{tabular}

$\mathrm{NASH}$, nonalcoholic steatohepatitis.

increased AST ( $\mathrm{P}=0.0318$; Table II). In addition, significant differences were identified in NASH-related pathological findings including steatosis, ballooning, inflammation, NASH stage and NASH grade in selected samples (NASH, $\mathrm{n}=6$; normal, $\mathrm{n}=7$ ). The grading of NASH-related pathological findings was more severe in the NASH model than in normal liver (Table III). 
Table IV. BAP and d-ROM values pre and post-PVE in normal liver.

\begin{tabular}{|c|c|c|}
\hline PVE & $0 W(n=6)$ & $2 W(n=6)$ \\
\hline \multicolumn{3}{|l|}{ BAP } \\
\hline Mean & $2,721.4$ & $2,792.7$ \\
\hline $\mathrm{SD}$ & 164.6 & 281.5 \\
\hline P-value & 0.604 & \\
\hline \multicolumn{3}{|l|}{ d-ROM } \\
\hline Mean & 165.3 & 289.1 \\
\hline SD & 37.8 & 130.8 \\
\hline P-value & 0.0501 & \\
\hline Sham & $0 W(n=5)$ & $2 W(n=5)$ \\
\hline \multicolumn{3}{|l|}{ BAP } \\
\hline Mean & $2,711.8$ & $2,656.5$ \\
\hline $\mathrm{SD}$ & 181.8 & 147.7 \\
\hline P-value & 0.6119 & \\
\hline \multicolumn{3}{|l|}{ d-ROM } \\
\hline Mean & 159.2 & 209.6 \\
\hline $\mathrm{SD}$ & 26.2 & 25.6 \\
\hline P-value & 0.0152 & \\
\hline
\end{tabular}

d-ROM, derivatives of reactive oxygen metabolites; BAP, biological antioxidative potential; PVE, portal vein embolization; W, weeks; $\mathrm{SD}$, standard deviation.

Table V. BAP and d-ROM values in NASH liver.

\begin{tabular}{lcc}
\hline NASH liver & 0W $(\mathrm{n}=11)$ & $4 \mathrm{~W}(\mathrm{n}=11)$ \\
\hline BAP & & \\
Mean & $2,856.1$ & 2361 \\
SD & 153.2 & 207.7 \\
P-value & $<0.0001$ & \\
d-ROM & & 182.8 \\
Mean & 203.0 & 45.0 \\
SD & 47.8 & \\
P-value & 0.3197 &
\end{tabular}

d-ROM, derivatives of reactive oxygen metabolites; BAP, biological antioxidative potential; NASH, nonalcoholic steatohepatitis; $\mathrm{W}$, weeks; SD, standard deviation.

Effects on BAP and d-ROM. Results of sequential changes in BAP and d-ROM values are summarized in Tables IV-VI.

Normal liver. BAP at 2 weeks after PVE was not significantly altered compared with that immediately prior to PVE $(\mathrm{P}=0.604)$. Likewise, the level of $\mathrm{d}-\mathrm{ROM}$ at 2 weeks after PVE was not significantly changed relative to that prior to PVE $(\mathrm{P}=0.0501$; Table IV).
Table VI. BAP and d-ROM values pre and post-PVE in NASH liver.

NASH liver

\begin{tabular}{lcc}
\hline PVE & $0 \mathrm{~W}(\mathrm{n}=5)$ & $2 \mathrm{~W}(\mathrm{n}=5)$ \\
\hline BAP & & \\
Mean & $2,496.9$ & $2,598.8$ \\
SD & 63.0 & 371.9 \\
P-value & 0.5625 & \\
d-ROM & & 396.8 \\
Mean & 168.4 & 49.4 \\
SD & 29.4 & \\
P-value & $<0.0001$ & $2 \mathrm{~W}(\mathrm{n}=6)$ \\
\hline Sham & $0 \mathrm{~W}(\mathrm{n}=6)$ & \\
\hline BAP & & $2,590.3$ \\
Mean & $2,472.9$ & 120.0 \\
SD & 202.0 & \\
P-value & 0.249 & 52.0 \\
d-ROM & & \\
Mean & 192.8 & \\
SD & 35.1 & \\
P-value & 0.0189 & \\
& &
\end{tabular}

d-ROM, derivatives of reactive oxygen metabolites; BAP, biological antioxidative potential; NASH, nonalcoholic steatohepatitis; PVE, portal vein embolization; $\mathrm{W}$, weeks; SD, standard deviation.

Sham. BAP at 2 weeks after no treatment was not significantly altered compared with that immediately prior to the Sham procedure $(\mathrm{P}=0.6119)$. Interestingly, the level of d-ROM at 2 weeks after no treatment was significantly higher than that detected immediately prior to Sham operation $(\mathrm{P}=0.0152$; Table IV).

NASH model. In the process of NASH model creation, BAP was significantly decreased $(\mathrm{P}<0.0001)$, but $\mathrm{d}-\mathrm{ROM}$ value was unchanged $(\mathrm{P}=0.3197$; Table $\mathrm{V})$.

$N A S H+P V E$. BAP at 7 weeks (2 weeks after PVE) was not significantly altered compared with that at 5 weeks $(\mathrm{P}=0.5625)$. The level of $\mathrm{d}-\mathrm{ROM}$ at 2 weeks after PVE was significantly elevated compared with that prior to PVE $(\mathrm{P}<0.0001$; Table VI).

$N A S H+S H A M$. BAP at 2 weeks after no PVE did not differ significantly to that detected prior to initiation of the high-fat $\operatorname{diet}(\mathrm{P}=0.249)$. The level of $\mathrm{d}-\mathrm{ROM}$ at 2 weeks after no PVE was significantly increased compared with that prior to starting the high-fat diet $(\mathrm{P}=0.0189$; Table VI).

\section{Discussion}

The present results indicated that PVE did not generate excessive d-ROM in normal liver Conversely, d-ROM increased in the Sham group. Meanwhile, PVE generated excessive d-ROM 
in NASH liver. It could therefore be concluded that PVE generates d-ROM production in NASH liver.

According to previous studies, PVE may produce oxidative stress through tumor necrosis factor- $\alpha$ (8-10) and interleukin-6 (10). Deposition of free fatty acids supposedly induces hepatocyte damage due to oxidative stress (11-14). On the basis of the current results, it appears a useful prospective application to focus on excessive oxidative stress when performing PVE in patients with NASH. In the current study, although liver regeneration could not be evaluated for the effect of PVE (since volumetric study of regenerated liver lobe without PVE was not performed), it may be speculated that excessive oxidative stress has some effect on the degree of liver regeneration or postoperative liver damage. To verify this hypothesis, further prospective experimental or clinical studies are warranted.

Although PVE did not reduce antioxidative potential in normal or NASH liver models, NASH itself reduced antioxidative potential. Several researchers have reported that reductions in antioxidative potential may serve a role in progression of NASH or metabolic syndrome $(11,15,16)$. On the basis of the current results, it may be proposed that the balance between oxidative and antioxidant stress serves a key role in the pathogenesis of NASH.

Interestingly, the level of d-ROM at 2 weeks after no treatment was significantly higher than that detected immediately prior to Sham operation. Although the exact reason for this is unclear, a certain level of stress due to Sham operation may occur.

Several limitations to the present study must be considered. First, there was a lack of data regarding hypertrophy of the non-embolized liver lobe and shrinkage of the embolized liver lobe following PVE. Second, measurement of d-ROM and BAP may have been somewhat variable in this study due to the chylous appearance of plasma samples. Standardization of measurements was therefore performed by skimming fatty components with PE prior to ordinary assays for BAP and d-ROM in the FRAS4. Third, there was no checking of sequential changes in liver volume pre and post-PVE. Thus the effects of PVE on liver tissue could not be objectively confirmed. Fourth, results of pathological examinations associated with NASH grade and score between normal and NASH groups were based on limited $(n=13)$ resected specimens. This incomplete pathological examination was attributed to unknown technical or other miscellaneous errors. Additionally, pathological specimens were examined 2 weeks after resuming a normal diet in the NASH group. Although it is uncertain whether the pathological changes associated with NASH are reversible after stopping intake of a high-fat diet, this change may have had some effect on the non-embolized liver lobe at the time of sacrifice. Fifth, neither high fat diet feeding nor PVE was compared with the matched controls.

In conclusion, the current experimental study determined that excessive oxidative stress may be generated following PVE in a NASH model. There must therefore be consideration of the risk of liver damage when performing PVE in NASH patients.

\section{Acknowledgements}

The authors would like to thank Associate Professor Ryozo Kamimura at the Institute of Laboratory Animal Science,
Kagoshima University, Kagoshima, Japan, for advising the method of anesthesia for rabbits.

\section{Funding}

Not applicable.

\section{Availability of data and materials}

All data generated or analyzed during this study are included in this published article.

\section{Authors' contributions}

YB, SH and TY designed the study. YB, SH and KN performed the surgical procedure. NT and SS measured d-ROM and BAP. MH performed the pathological examination of resected tissue. YB, SH and TY wrote the manuscript. All authors read and approved the final version of the manuscript.

\section{Ethics approval and consent to participate}

All animal procedures were conducted via a protocol approved by the Kagoshima University of Science Institutional Animal Care and Use Committee (Kagoshima, Japan; approval no. MD11117).

\section{Consent for publication}

Not applicable.

\section{Competing interests}

The authors declare that they have no competing interests.

\section{References}

1. Baba Y, Sonoda JI, Hayashi S, Tosuji N, Sonoda S, Makisumi K and Nakajo M: Reduction of oxidative stress in liver cancer patients by oral green tea polyphenol tablets during hepatic arterial infusion chemotherapy. Exp Ther Med 4: 452-458, 2012.

2. Yokoyama Y, Nagino M and Nimura Y: Mechanisms of hepatic regeneration following portal vein embolization and partial hepatectomy: A review. World J Surg 31: 367-374, 2007.

3. Khan AZ, Morris-Stiff G and Makuuchi M: Patterns of chemotherapy-induced hepatic injury and their implications for patients undergoing liver resection for colorectal liver metastases. J Hepatobiliary Pancreat Surg 16: 137-144, 2009.

4. Narita M, Oussoultzoglou E, Chenard MP, Rosso E, Casnedi S, Pessaux P, Bachellier P and Jaeck D: Sinusoidal obstruction syndrome compromises liver regeneration in patients undergoing two-stage hepatectomy with portal vein embolization. Surg Today 41: 7-17, 2011.

5. Usuki N: Selective segmental necrotizing therapy for hepatocellular carcinoma: An experimental study of an ethanol injection method. Nihon Igaku Hoshasen Gakkai Zasshi 54: 1142-1154, 1994 (In Japanese).

6. Fu JF, Fang YL, Liang L, Wang CL, Hong F and Dong GP: A rabbit model of pediatric nonalcoholic steatohepatitis: The role of adiponectin. World J Gastroenterol 15: 912-918, 2009.

7. Brunt EM, Janney CG, Di Bisceglie AM, Neuschwander-Tetri BA and Bacon BR: Nonalcoholic steatohepatitis: A proposal for grading and staging the histological lesions. Am J Gastroenterol 94: 2467-2474, 1999

8. Sutti S, Jindal A, Locatelli I, Vacchiano M, Gigliotti L, Bozzola C and Albano E: Adaptive immune responses triggered by oxidative stress contribute to hepatic inflammation in NASH. Hepatology 59: 886-897, 2014. 
9. Ypsilantis P, Lambropoulou M, Anagnostopoulos C, Tentes I, Tsigalou C, Pitiakoudis M, Kortsaris A, Papadopoulos N and Simopoulos C: Mesna preserves hepatocyte regenerating capacity following liver radiofrequency ablation under Pringle maneuver. J Surg Res 169: 44-50, 2011.

10. Handa P, Morgan-Stevenson V, Maliken BD, Nelson JE, Washington S, Westerman M, Yeh MM and Kowdley KV: Iron overload results in hepatic oxidative stress, immune cell activation, and hepatocellular ballooning injury, leading to nonalcoholic steatohepatitis in genetically obese mice. Am J Physiol Gastrointest Liver Physiol 310: G117-G127, 2016.

11. Furukawa S, Fujita T, Shimabukuro M, Iwaki M, Yamada Y, Nakajima Y, Nakayama O, Makishima M, Matsuda M and Shimomura I: Increased oxidative stress in obesity and its impact on metabolic syndrome. J Clin Invest 114: 1752-1761, 2004.

12. Vecchione G, Grasselli E, Compalati AD, Ragazzoni M, Cortese K, Gallo G, Voci A and Vergani L: Ethanol and fatty acids impair lipid homeostasis in an in vitro model of hepatic steatosis. Food Chem Toxicol 90: 84-94, 2016.
13. Liu W, Baker RD, Bhatia T, Zhu L and Baker SS: Pathogenesis of nonalcoholic steatohepatitis. Cell Mol Life Sci 73: 1969-1987, 2016.

14. Li S, Zeng XY, Zhou X, Wang H, Jo E, Robinson SR, Xu A and Ye JM: Dietary cholesterol induces hepatic inflammation and blunts mitochondrial function in the liver of high-fat-fed mice. J Nutr Biochem 27: 96-103, 2016.

15. Kitade $\mathrm{H}$, Chen $\mathrm{G}, \mathrm{Ni}$ Y and Ota T: Nonalcoholic fatty liver disease and insulin resistance: New insights and potential new treatments. Nutrients 9: E387, 2017.

16. Richter K and Kietzmann T: Reactive oxygen species and fibrosis: Further evidence of a significant liaison. Cell Tissue Res 365: 591-605, 2016. 